\title{
Characterization of tomographically faithful states in terms of their Wigner function
}

\author{
G M D'Ariano $\S$ and M F Sacchi \\ QUIT, INFM and Dipartimento di Fisica "A. Volta", Università di Pavia, via A. \\ Bassi 6, I-27100 Pavia, Italy
}

\begin{abstract}
A bipartite quantum state is tomographically faithful when it can be used as an input of a quantum operation on one of the two quantum systems, such that the joint output state carries a complete information about the operation itself. Tomographically faithful states are a necessary ingredient for tomography of quantum operations and for complete quantum calibration of measuring apparatuses. In this paper we provide a complete classification of such states for continuous variables in terms of the Wigner function of the state. For two-mode Gaussian states faithfulness simply resorts to correlation between the modes.

PACS numbers: 03.65.Wj
\end{abstract}

\section{Introduction}

Quantum operations describe any kind of physical process affecting quantum states, including unitary evolutions of closed systems and non unitary transformations of open quantum systems, such as systems interacting with a reservoir, or subjected to noise or measurements of any kind. The problem of determining experimentally the quantum operation occurs in different scenarios, typically for quantum calibration of controlled transformations [1] and of measuring apparatuses [2].

In a naive process tomography one varies the input state over a suitably complete set in order to recover enough information about the quantum operation. The tensor structure of the bipartite quantum system, however, allows to use a single fixed bipartite state that scans the complete set of single-system states in a quantum parallel fashion [1]. The bipartite states that can be used in this way in order to carry a complete information of the process are called tomographically faithful 3 .

The problem of evaluating the faithfulness of a state can be expressed in terms of an invertibility condition of a map associated to the state. In many situations it is not simple to check such a condition. In this paper we address the continuous variables case (i.e. quantum harmonic oscillators), and solve the problem of the complete classification of faithfulness in terms of the Wigner function of the state. The use of Wigner functions has proved very useful as a generalized phase-space technique to $\S$ To whom correspondence should be addressed. 
express density operators in terms of $c$-number functions, thus leading to a considerable simplification of the evaluation of quantum dynamics and of expectation values [4, 5].

In this paper we present a general result that provides a necessary and sufficient condition for the faithfulness in terms of Wigner function. Such a condition, as we will show, makes use of highly irregular functions, as the customary $P$-functions in quantum optics. We then specialize our results to the case of Gaussian states of two modes of the electromagnetic field. The class of Gaussian states constitutes a fortunate framework both for theoreticians and experimentalists, since, on one side all calculations can be done analytically, whereas, on the other side, these states are easily generated in a lab, using lasers, linear optics, and parametric amplifiers. We will show that for Gaussian states the condition of faithfulness is just the existence of correlations between the two modes.

The paper is organized as follows. In Sec. II we briefly recover the general result about the faithfulness of a quantum state, and recall the problem of inversion of a special operator associated to the state. We then restate the problem in terms of Wigner function of the state, and write a necessary and sufficient condition. The section presents some examples of faithful (both entangled and separable) and unfaithful states. In Sec. III we simplify the result of Sec. II for the case of two-mode Gaussian states. The condition of faithfulness then simply restates as the existence of correlations between the two modes. We conclude the paper in Sec. IV, with a summary of results and some remarks about the statistical errors that affect the reconstruction of a quantum operation, and the connection with quantum images.

\section{Faithfulness in terms of Wigner function}

In mathematical terms, a quantum operation $\mathcal{E}$ is described by a completely positive map [6]. This can be written in the Kraus form

$$
\mathcal{E}(\rho)=\sum_{n} K_{n} \rho K_{n}^{\dagger}
$$

where $K_{n}$ are operators on the Hilbert space $\mathcal{H}$ of the quantum system. For simplicity we will consider quantum operations with the same input and output space $\mathcal{H}$, and that are trace-preserving - the so-called channels - corresponding to the completeness

relation $\sum_{n} K_{n}^{\dagger} K_{n}=I$. The concept of tomographically faithful state [3] relies on using a bipartite state $R$ on $\mathcal{H} \otimes \mathcal{H}$, such that the output state

$$
R_{\mathcal{E}}=(\mathcal{E} \otimes I) R
$$

is in one-to-one correspondence to the quantum operation $\mathcal{E}$. In 3 it was proved that a state $R$ is faithful iff the following operator on $\mathcal{H} \otimes \mathcal{H}$

$$
\check{R}=(E R)^{\tau_{2}} E=\left(R^{\tau_{2}} E\right)^{\tau_{1}}
$$

is invertible. In Eq. (3) $E=\sum_{i, j}|i j\rangle\langle j i|$ denotes the swap operator, and $O^{\tau_{l}}$ denotes the partial transposition of the operator $O$ on the $l$ th Hilbert space, $l=1,2$. 
Using the notation of Ref. [7] for bipartite vectors

$$
|A\rangle\rangle \equiv \sum_{n, m}\langle n|A| m\rangle|n\rangle \otimes|m\rangle,
$$

one can generally write a bipartite state in the form

$$
\left.R=\sum_{i, j}\left|A_{i}\right\rangle\right\rangle\left\langle\left\langle B_{j}\right| .\right.
$$

From the identity

$$
\left.A \otimes B|C\rangle\rangle=\left|A C B^{\tau}\right\rangle\right\rangle
$$

it follows that

$$
\check{R}=\sum_{i, j} A_{i}^{\tau} \otimes B_{j}^{\dagger}
$$

where the transpose $\tau$ is defined on the basis chosen in the decomposition of Eq. (4). Similarly, for a state $R$ written as

$$
R=\sum_{i, j} A_{i} \otimes B_{j},
$$

using Eq. (3), one easily writes $\check{R}$ as follows

$$
\left.\check{R}=\sum_{i, j}\left|B_{j}\right\rangle\right\rangle\left\langle\left\langle A_{i}^{*}\right|,\right.
$$

where $O^{*}$ denotes the complex conjugation on the fixed basis. Notice that the evaluation the operator $\check{R}$ does not need the diagonalization of $R$. Moreover, all the previous sums can be suitably replaced with integrals.

A state $R$ is faithful iff the associated operator $\check{R}$ is invertible. We are interested in finding the conditions of faithfulness in terms of the Wigner function of the state. For simplicity, we consider bipartite states that correspond to two-mode states. However, our results are easily generalized to the case of two-party multimode states.

We recall the Cahill-Glauber formulas [8] between single-mode density matrix $\rho$ and Wigner function $W\left(\alpha, \alpha^{*}\right)$

$$
\begin{aligned}
& W\left(\alpha, \alpha^{*}\right)=\frac{2}{\pi} \operatorname{Tr}\left[\rho D(2 \alpha)(-1)^{a^{\dagger} a}\right], \\
& \rho=2 \int_{\mathbb{C}} d^{2} \alpha W\left(\alpha, \alpha^{*}\right) D(2 \alpha)(-1)^{a^{\dagger} a},
\end{aligned}
$$

where $\alpha^{*}$ denotes the complex conjugate of $\alpha, d^{2} \alpha \equiv d \operatorname{Re}(\alpha) d \operatorname{Im}(\alpha)$, and $D(\alpha)=$ $e^{\alpha a^{\dagger}-\alpha^{*} a}$ represents the displacement operator for the mode $a$, with $\left[a, a^{\dagger}\right]=1$.

For a two-mode bipartite state $R$ we write the Wigner function as a function of two complex variables $\alpha$ and $\beta$ by direct generalization of Eq. (11) as follows

$$
R=4 \int_{\mathbb{C}} d^{2} \alpha \int_{\mathbb{C}} d^{2} \beta W\left(\alpha, \alpha^{*}, \beta, \beta^{*}\right) D(2 \alpha)(-1)^{a^{\dagger} a} \otimes D(2 \beta)(-1)^{b^{\dagger} b},
$$

where $a$ is a shorthand notation for the annihilation operator otherwise denoted $a \otimes I$, as well as $b$ for $I \otimes b$. In the following we will write the Wigner function shortly 
Characterization of tomographically faithful states in terms of their Wigner function 4 as $W(\alpha, \beta) \equiv W\left(\alpha, \alpha^{*}, \beta, \beta^{*}\right)$, omitting the dependence on the complex conjugated variables. According to Eqs. (8) and (9), the condition of faithfulness of the state in (12) corresponds to the condition of invertibility of the operator

$$
\begin{aligned}
\check{R} & \left.=4 \int_{\mathbb{C}} d^{2} \alpha \int_{\mathbb{C}} d^{2} \beta W(\alpha, \beta)\left|D(2 \beta)(-1)^{a^{\dagger} a}\right\rangle\right\rangle\left\langle\left\langle D\left(2 \alpha^{*}\right)(-1)^{a^{\dagger} a}\right|\right. \\
& \left.=4\left(I \otimes(-1)^{b^{\dagger} b}\right) \int_{\mathbb{C}} d^{2} \alpha \int_{\mathbb{C}} d^{2} \beta W(\alpha, \beta)|D(2 \beta)\rangle\right\rangle\left\langle\left\langle D\left(2 \alpha^{*}\right)\right|\left(I \otimes(-1)^{b^{\dagger} b}\right),\right.
\end{aligned}
$$

where in the second line we used identity ([6). Since the set $\{|D(\alpha)\rangle\rangle\}$ is an orthonormal basis (in the Dirac sense) for $\mathcal{H} \otimes \mathcal{H}$, namely $\left.\int_{\mathbb{C}} \frac{d^{2} \alpha}{\pi}|D(\alpha)\rangle\right\rangle\langle\langle D(\alpha)|=I \otimes I$, the condition of faithfulness in terms of Wigner function is then the following:

Necessary and sufficient condition for faithfulness: a bipartite state with Wigner function $W(\alpha, \beta)$ is faithful iff one can find a function $f(\beta, \gamma)$ such that

$$
\int_{\mathbb{C}} d^{2} \beta W(\alpha, \beta) f(\beta, \gamma)=\delta^{(2)}(\alpha-\gamma),
$$

where $\delta^{(2)}(\sigma)=\int_{\mathbb{C}} \frac{d^{2} \lambda}{\pi^{2}} e^{\lambda \sigma^{*}-\lambda^{*} \sigma}$ denotes the Dirac delta over the complex plane.

Equation (14) should be read in distributional sense. When such a condition is satisfied one has

$$
\left.\check{R}^{-1}=\frac{4}{\pi}\left(I \otimes(-1)^{b^{\dagger} b}\right) \int_{\mathbb{C}} d^{2} \delta \int_{\mathbb{C}} d^{2} \gamma f(\delta, \gamma)\left|D\left(2 \gamma^{*}\right)\right\rangle\right\rangle\left\langle\langle D(2 \delta)|\left(I \otimes(-1)^{b^{\dagger} b}\right) .\right.
$$

\subsection{Example 1: twin-beam state}

Consider the twin-beam state that can be easily generated by nondegenerate optical parametric amplifiers

$$
R=\left(1-\lambda^{2}\right)\left|\lambda^{a^{\dagger} a}\right\rangle\left\langle\left\langle\lambda^{a^{\dagger} a}\right|, \quad 0 \leq \lambda<1,\right.
$$

where the parameter $\lambda$ is simply related to the total number of photons $\bar{n}=2 \lambda^{2} /\left(1-\lambda^{2}\right)$. The corresponding Wigner function is given by

$$
W_{R}(\alpha, \beta)=\frac{4\left(1-\lambda^{2}\right)}{\pi^{2}} \operatorname{Tr}\left[\lambda^{a^{\dagger} a} D(2 \alpha) \lambda^{a^{\dagger} a} D\left(-2 \beta^{*}\right)\right] .
$$

By normal ordering Eq. (17) and a lengthy calculation, one obtains

$$
W_{R}(\alpha, \beta)=\frac{4}{\pi^{2}} \exp \left[-\frac{2\left(1+\lambda^{2}\right)}{1-\lambda^{2}}\left(|\alpha|^{2}+|\beta|^{2}\right)+\frac{4 \lambda}{1-\lambda^{2}}\left(\alpha \beta+\alpha^{*} \beta^{*}\right)\right] .
$$

Using the solution (41) of identity (14) derived in the appendix, the function $f(\beta, \gamma)$ can formally be written as

$$
f(\beta, \gamma)=\frac{4 \lambda^{2}}{\pi\left(1-\lambda^{2}\right)^{2}} e^{\frac{1+3 \lambda^{2}}{1-\lambda^{2}}|\beta|^{2}} e^{2 \frac{1-8 \lambda^{2}+\lambda^{4}}{\left(1-\lambda^{2}\right)^{2}}|\gamma|^{2}} \int_{\mathbb{C}} d^{2} \xi e^{|\xi|^{2}} e^{\xi\left(\frac{4 \lambda}{1-\lambda^{2}} \gamma-\beta^{*}\right)-\xi^{*}\left(\frac{4 \lambda}{1-\lambda^{2}} \gamma^{*}-\beta\right)},
$$

which should be treated as a distribution, in the sense that the integral in $\xi$ has to be performed after the integration on $\beta$ of Eq. (14). 
Notice that in this simple example, the faithfulness is more easily checked using Eq. (77) and writing immediately

$$
\check{R}^{-1}=\frac{1}{1-\lambda^{2}}\left(\frac{1}{\lambda}\right)^{a^{\dagger} a} \otimes\left(\frac{1}{\lambda}\right)^{b^{\dagger} b} .
$$

\subsection{Example 2: classically correlated coherent states}

A mixture of correlated coherent states can be easily generated by splitting thermal radiation in a 50/50 beam splitter. For such a kind of states we can write

$$
R=\int_{\mathbb{C}} \frac{d^{2} \gamma}{\pi \sigma^{2}} e^{-\frac{|\gamma|^{2}}{\sigma^{2}}}|\gamma\rangle\left\langle\left.\gamma\right|^{\otimes 2}\right.
$$

where the variance $\sigma$ is related to the total number of photons by $\bar{n}=\sigma / 2$. The corresponding Wigner function is given by

$$
W_{R}(\alpha, \beta)=\frac{4}{\pi^{2}\left(1+2 \sigma^{2}\right)} \exp \left[-\frac{2}{1+2 \sigma^{2}}\left(|\alpha|^{2}+|\beta|^{2}\right)+\frac{4 \sigma^{2}}{1+2 \sigma^{2}}\left(\alpha \beta^{*}+\alpha^{*} \beta\right)\right] .
$$

Using again the solution (41) of identity (14) derived in the appendix, the function $f(\beta, \gamma)$ can formally be written as

$$
f(\beta, \gamma)=\frac{4 \sigma^{4}}{\pi\left(1+2 \sigma^{2}\right)} e^{\frac{1-2 \sigma^{2}}{1+2 \sigma^{2}}|\beta|^{2}} e^{2 \frac{1+2 \sigma^{2}-8 \sigma^{4}}{\left(1+2 \sigma^{2}\right)^{2}}|\gamma|^{2}} \int_{\mathbb{C}} d^{2} \xi e^{|\xi|^{2}} e^{\xi\left(\beta-\frac{4 \sigma^{2}}{1+2 \sigma^{2}} \gamma\right)-\xi^{*}\left(\beta^{*}-\frac{4 \sigma^{2}}{1+2 \sigma^{2}} \gamma^{*}\right)},
$$

and thus the state (21) is an example of separable faithful state.

\subsection{Example 3: product states}

Consider a product state

$$
R=\rho \otimes \sigma .
$$

The Wigner function is given by the product of the independent Wigner functions for $\rho$ and $\sigma$

$$
W_{R}(\alpha, \beta)=W_{\rho}\left(\alpha, \alpha^{*}\right) W_{\sigma}\left(\beta, \beta^{*}\right) .
$$

Of course the state $R$ is not faithful, and in fact the condition (14) can never be satisfied.

\subsection{Example 4: classical correlation between orthogonal states}

Consider the state

$$
R=(1-\lambda) \sum_{n=0}^{\infty} \lambda^{n}|n\rangle\left\langle\left. n\right|^{\otimes 2},\right.
$$

where $|n\rangle$ denotes the Fock state. From the relation [8]

$$
\langle n|D(\alpha)| n\rangle=e^{-\frac{|\alpha|^{2}}{2}} L_{n}\left(|\alpha|^{2}\right)
$$

and the identity [9]

$$
\sum_{n=0}^{\infty} \lambda^{n} L_{n}(x) L_{n}(y)=\frac{1}{1-\lambda} e^{-\frac{\lambda}{1-\lambda}(x+y)} I_{0}\left(2 \frac{\sqrt{x y \lambda}}{1-\lambda}\right)
$$


where $L_{n}(x)$ and $I_{0}(x)$ denote the $n$-th order Laguerre polynomials and the 0 -order modified Bessel function, one obtains the Wigner function

$$
W_{R}(\alpha, \beta)=\frac{4}{\pi^{2}} e^{-2 \frac{1+\lambda}{1-\lambda}\left(|\alpha|^{2}+|\beta|^{2}\right)} I_{0}\left(\frac{8 \sqrt{\lambda}}{1-\lambda}|\alpha \beta|\right) .
$$

Condition (14) can never be satisfied, since there is no dependence of the Wigner function on the phase of $\beta$. In fact, $\check{R} \equiv R$ is clearly not invertible, whence the state $R$ is not faithful.

\section{Simplification for Gaussian states}

Unfortunately, it is often difficult to inspect condition (14), since it holds more generally in a distribution sense. For Gaussian bipartite states, however, it is possible to derive a more practical condition in terms of the correlation matrix.

According to the derivation in the appendix, the term of the Wigner function of a Gaussian bipartite state that is relevant for the condition (14) is the factor of the form

$$
g(\alpha, \beta)=\exp \left[\left(A \alpha \beta+B \alpha \beta^{*}\right)+\text { h.c. }\right],
$$

In fact, as shown in the appendix, the condition (14) can be satisfied iff

$$
|A|^{2}-|B|^{2} \neq 0 \text {. }
$$

In order to clarify the meaning of condition (31), it is useful to consider the state $R$ in terms of the characteristic function $\Gamma(\alpha, \beta)=\operatorname{Tr}[R D(\alpha) \otimes D(\beta)]$, that corresponds to the Fourier transform of the Wigner function, and hence will be Gaussian as well. One has

$$
R=\int_{\mathbb{C}} \frac{d^{2} \alpha}{\pi} \int_{\mathbb{C}} \frac{d^{2} \beta}{\pi} \Gamma(\alpha, \beta) D^{\dagger}(\alpha) \otimes D^{\dagger}(\beta) .
$$

The operator $\check{R}$ then can be written as

$$
\left.\check{R}=\int_{\mathbb{C}} \frac{d^{2} \alpha}{\pi} \int_{\mathbb{C}} \frac{d^{2} \beta}{\pi} \Gamma(\alpha, \beta)\left|D^{\dagger}(\beta)\right\rangle\right\rangle\left\langle\left\langle D^{\dagger}\left(\alpha^{*}\right)\right| .\right.
$$

Similarly to Eq. (14), $\check{R}$ is invertible iff one can find a function $f(\beta, \gamma)$ such that

$$
\int_{\mathbb{C}} d^{2} \beta \Gamma(\alpha, \beta) f(\beta, \gamma)=\delta^{(2)}(\alpha-\gamma)
$$

and $\check{R}^{-1}$ can be written as

$$
\left.\check{R}^{-1}=\int_{\mathbb{C}} d^{2} \delta \int_{\mathbb{C}} d^{2} \gamma f(\delta, \gamma)\left|D^{\dagger}\left(\gamma^{*}\right)\right\rangle\right\rangle\left\langle\left\langle D^{\dagger}(\delta)\right| .\right.
$$

The same consideration before Eq. (30) applies here. The condition (34) can be satisfied iff $|A|^{2}-|B|^{2} \neq 0$, where $A$ and $B$ are the coefficients in front of the variables $\alpha \beta$ and $\alpha \beta^{*}$ of the characteristic function. Using the identities

$$
\begin{aligned}
& A=\left.\partial_{\alpha \beta}^{2} \Gamma(\alpha, \beta)\right|_{\alpha=\beta=0}-\left.\left.\partial_{\alpha} \Gamma(\alpha, \beta)\right|_{\alpha=\beta=0} \partial_{\beta} \Gamma(\alpha, \beta)\right|_{\alpha=\beta=0} \\
& A^{*}=\left.\partial_{\alpha^{*} \beta^{*}}^{2} \Gamma(\alpha, \beta)\right|_{\alpha=\beta=0}-\left.\left.\partial_{\alpha^{*}} \Gamma(\alpha, \beta)\right|_{\alpha=\beta=0} \partial_{\beta^{*}} \Gamma(\alpha, \beta)\right|_{\alpha=\beta=0} \\
& B=\left.\partial_{\alpha \beta^{*}}^{2} \Gamma(\alpha, \beta)\right|_{\alpha=\beta=0}-\left.\left.\partial_{\alpha} \Gamma(\alpha, \beta)\right|_{\alpha=\beta=0} \partial_{\beta^{*}} \Gamma(\alpha, \beta)\right|_{\alpha=\beta=0} \\
& B^{*}=\left.\partial_{\alpha^{*} \beta}^{2} \Gamma(\alpha, \beta)\right|_{\alpha=\beta=0}-\left.\left.\partial_{\alpha^{*}} \Gamma(\alpha, \beta)\right|_{\alpha=\beta=0} \partial_{\beta} \Gamma(\alpha, \beta)\right|_{\alpha=\beta=0},
\end{aligned}
$$


the condition of faithfulness can be restated in terms of the correlation matrix as follows:

Necessary and sufficient condition for faithfulness of Gaussian states: a bipartite Gaussian state is faithful iff the following condition on the correlations is satisfied

$$
\chi(R) \doteq\left\langle\Delta a^{\dagger} b^{\dagger}\right\rangle\langle\Delta a b\rangle+\left\langle\Delta a^{\dagger} b\right\rangle\left\langle\Delta a b^{\dagger}\right\rangle \neq 0,
$$

where for any two operators $P$ and $Q$

$$
\langle\triangle P Q\rangle \doteq\langle P Q\rangle-\langle P\rangle\langle Q\rangle \text {. }
$$

In terms of the quadratures $X_{c}=\left(c+c^{\dagger}\right) / 2$ and $Y_{c}=\left(c-c^{\dagger}\right) /(2 i)$ of the modes $c=a, b$, the correlation $\chi(R)$ can be rewritten as

$$
\chi(R)=\frac{1}{2}\left(\left\langle\Delta X_{a} X_{b}\right\rangle^{2}+\left\langle\Delta Y_{a} Y_{b}\right\rangle^{2}+\left\langle\Delta X_{a} Y_{b}\right\rangle^{2}+\left\langle\Delta Y_{a} X_{b}\right\rangle^{2}\right) .
$$

In the examples $1-4$ given in the previous section, one has $\chi(R)=\frac{\lambda^{2}}{\left(1-\lambda^{2}\right)^{2}}, \sigma^{4}, 0$, and 0 , respectively (notice, however, that the state in example 4 is not Gaussian). Using Eq. (39) the condition $\chi(R) \neq 0$ shows that bipartite Gaussian states are always faithful, except when they are product states $(\chi(R)$ is the sum of nonnegative terms which all vanish when there is no correlation between the two modes). A rigorous relation bewteen statistical errors that affect tomographic reconstructions and the strength of correlations is in order, but beyond the scope of this paper.

\section{Conclusions}

Tomographically faithful states are a necessary ingredient for tomography of quantum operations and for complete quantum calibration of measuring apparatuses. In this paper we have provided a complete classification of two-mode faithful states in terms of the Wigner function of the state. This classification has been derived from the general faithfulness condition resorting to the invertibility of a special operator associated to the state. Some examples of faithful states have been presented, both entangled and separable, along with examples of not faithful states. For two-mode Gaussian states we have shown that faithfulness is simply equivalent to nonvanishing correlations between the modes.

We conclude by noticing that the actual statistical efficiency of a faithful state in the tomography of a quantum operation in infinite dimensions is connected to the increase of the singular values of the unbounded operator $\check{R}^{-1}$. Such unboundedness is responsible of the increasingly large statistical errors in the Fock representation of the quantum operation, accounting for the finite experimental data sample used to infer information on a infinite set of matrix elements of the quantum operation. As a rule of thumb, the statistical efficiency increases for greater correlation $\chi(R)$.

Finally, it is worth mentioning that the framework of quantum images [10] bears a strict analogy with that of the quantum tomography of a channel using an input 
Characterization of tomographically faithful states in terms of their Wigner function 8

bipartite state, with the role of the channel here played by the density contour of the image analyzed by one of the twin beams from parametric downconversion of vacuum. Clearly, when the state is faithful one has quantum imaging on the other beam, and our result is consistent with the recent demonstration [10] that entanglement is not necessary for quantum imaging. In particular, the thermal state split by a beam splitter in Eq. (21) is suitable for quantum imaging.

\section{Acknowledgments}

This work has been sponsored by INFM through the project PRA-2002-CLON, and by EC and MIUR through the cosponsored ATESIT project IST-2000-29681 and Cofinanziamento 2003.

\section{Appendix}

We show that for a function of the form

$$
g(\alpha, \beta)=h(\alpha) k(\beta) e^{A \alpha \beta+A^{*} \alpha^{*} \beta^{*}} e^{B \alpha \beta^{*}+B^{*} \alpha^{*} \beta},
$$

with $|A| \neq|B|$, and both $h$ and $k$ (generally not analitycal) invertible functions, the following function

$$
\begin{aligned}
& f(\beta, \gamma)=\frac{\left(|A|^{2}-|B|^{2}\right)^{2}}{\pi^{3}} k^{(-1)}(\beta) h^{(-1)}(\gamma) e^{-\left(|A|^{2}+|B|^{2}\right)|\gamma|^{2}-\left(A B \gamma^{2}+A^{*} B^{*} \gamma^{* 2}\right)} e^{-|\beta|^{2}} \\
& \times \int d^{2} \xi e^{\left(|A|^{2}+|B|^{2}\right)|\xi|^{2}+\left(A^{*} B^{*} \xi^{2}+A B \xi^{* 2}\right)} e^{\left(|A|^{2}-|B|^{2}\right)\left(\xi \gamma-\xi^{*} \gamma^{*}\right)} e^{\beta\left(A \xi^{*}+B^{*} \xi\right)-\beta^{*}\left(A^{*} \xi+B \xi^{*}\right)}
\end{aligned}
$$

satisfies the identity

$$
\int_{\mathbb{C}} d^{2} \beta g(\alpha, \beta) f(\beta, \gamma)=\delta^{(2)}(\alpha-\gamma) .
$$

The function $f(\beta, \gamma)$ should be treated as a distribution, in the sense that the integral in $\xi$ has to be performed after the integration on $\beta$ of Eq. (42).

One has

$$
\begin{aligned}
& \int_{\mathbb{C}} d^{2} \beta g(\alpha, \beta) f(\beta, \gamma)=\frac{\left(|A|^{2}-|B|^{2}\right)^{2}}{\pi^{3}} h(\alpha) h^{(-1)}(\gamma) e^{-\left(|A|^{2}+|B|^{2}\right)|\gamma|^{2}-\left(A B \gamma^{2}+A^{*} B^{*} \gamma^{* 2}\right)} \\
& \times \int d^{2} \xi e^{\left(|A|^{2}+|B|^{2}\right)|\xi|^{2}+\left(A^{*} B^{*} \xi^{2}+A B \xi^{* 2}\right)} e^{\left(|A|^{2}-|B|^{2}\right)\left(\xi \gamma-\xi^{*} \gamma^{*}\right)} \\
& \times \int d^{2} \beta e^{-|\beta|^{2}} e^{\beta\left(A \xi^{*}+B^{*} \xi+A \alpha+B^{*} \alpha^{*}\right)-\beta^{*}\left(A^{*} \xi+B \xi^{*}-A^{*} \alpha^{*}-B \alpha\right)} \\
& =\frac{\left(|A|^{2}-|B|^{2}\right)^{2}}{\pi^{2}} h(\alpha) h^{(-1)}(\gamma) e^{\left(|A|^{2}+|B|^{2}\right)\left(|\alpha|^{2}-|\gamma|^{2}\right)+A B\left(\alpha^{2}-\gamma^{2}\right)+A^{*} B^{*}\left(\alpha^{* 2}-\gamma^{* 2}\right)} \\
& \times \int d^{2} \xi e^{\left(|A|^{2}-|B|^{2}\right)\left[\xi(\gamma-\alpha)-\xi^{*}\left(\gamma^{*}-\alpha^{*}\right)\right]} \\
& =\delta^{(2)}(\alpha-\gamma)
\end{aligned}
$$


where the integral in $d^{2} \beta$ has been performed by using the identity

$$
\int_{\mathbb{C}} d^{2} \beta e^{-\frac{|\beta|^{2}}{\sigma^{2}}} e^{\beta \alpha^{*}-\beta^{*} \gamma}=\pi \sigma^{2} e^{-\sigma^{2} \alpha^{*} \gamma} .
$$

\section{References}

[1] G. M. D'Ariano and P. Lo Presti, Phys. Rev. Lett. 86, 4195 (2001)

[2] G. M. D'Ariano, P. Lo Presti, and L. Maccone, Phys. Rev. Lett. 93, 250407 (2004).

[3] G. M. D'Ariano and P. Lo Presti, Phys. Rev. Lett. 91, 047902 (2003).

[4] The Physics of Phase Space, edited by Y. S. Kim and W. W. Zachary (Springer, Berlin, 1986).

[5] C. W. Gardiner, Quantum Noise (Springer, Berlin, 1991).

[6] K. Kraus, States, Effects and Operations, Lecture Notes in Physics Vol. 190 (Springer, Berlin, 1983).

[7] G. M. D'Ariano, P. Lo Presti, and M. F. Sacchi, Phys. Lett. 272, 32 (2000).

[8] K. E. Cahill and R. J. Glauber, Phys. Rev. 177, 1857 (1969).

[9] I. S. Gradstein and I. M. Ryzhik, Table of Integrals, Series, and Products (Academic Press, New York, 1980).

[10] D. Magatti, F. Ferri, A. Gatti, M. Bache, E. Brambilla, and L. A. Lugiato, Phys. Rev. Lett. 94, $183602(2005)$. 